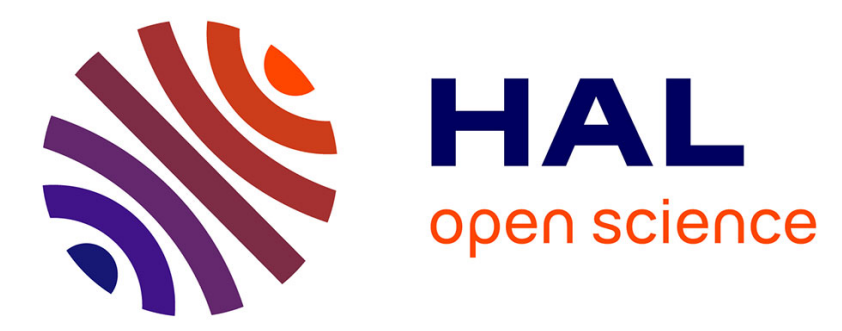

\title{
Utilisation d'un groupe nominal de consensus en institut de formation en soins infirmiers: un éclairage sur la qualité de vie des étudiants
}

\author{
Barbara Joannot, Yannick Dauge, Jean-Louis Boutte
}

\section{- To cite this version:}

Barbara Joannot, Yannick Dauge, Jean-Louis Boutte. Utilisation d'un groupe nominal de consensus en institut de formation en soins infirmiers: un éclairage sur la qualité de vie des étudiants. Pédagogie médicale, 2019, 10.1051/pmed/2019023 . hal-02395247

\section{HAL Id: hal-02395247 \\ https://hal.science/hal-02395247}

Submitted on 5 Dec 2019

HAL is a multi-disciplinary open access archive for the deposit and dissemination of scientific research documents, whether they are published or not. The documents may come from teaching and research institutions in France or abroad, or from public or private research centers.
L'archive ouverte pluridisciplinaire HAL, est destinée au dépôt et à la diffusion de documents scientifiques de niveau recherche, publiés ou non, émanant des établissements d'enseignement et de recherche français ou étrangers, des laboratoires publics ou privés. 


\title{
Utilisation d'un groupe nominal de consensus en institut de formation en soins infirmiers: un éclairage sur la qualité de vie des étudiants
}

\section{Use of a nominal consensus group in a Nursing Care Training Institute: an insight into the quality of life of students}

Barbara JOANNOT ${ }^{1, *}$, Yannick DAUGE ${ }^{2}$, et Jean-Louis BOUTTE ${ }^{3}$

${ }^{1}$ Institut de formation en ergothérapie, Pôle régional d'enseignement et de formation aux métiers de la santé (PREFMS), Toulouse, France

${ }^{2}$ Institut de formation en soins infirmiers, Groupement de coopération sanitaire du Pays d'Aix (GCSPA), Aix-en-Provence, France

${ }^{3}$ Département des sciences de l'éducation, Aix Marseille Université, Aix-en-Provence, France

Manuscrit soumis à la rédaction le 8 mai 2018 ; commentaires éditoriaux formulés aux auteurs le 20 octobre 2018 ; accepté pour publication le 13 septembre 2019

Résumé - Contexte: La situation des étudiants en soins infirmiers (ESI) de première année induit de nombreux changements dans plusieurs sphères d'activités, soient-ils personnels, familiaux ou scolaires, demandant à l'étudiant une adaptation rapide, pouvant modifier son équilibre et affecter sa qualité de vie $(\mathrm{QdV})$. But: L'objectif était d'identifier, selon ces étudiants, principaux concernés par cette notion multidimensionnelle et considérés comme expert de leur QdV, les facteurs susceptibles de l'influencer. Méthode: Un groupe de 7 ESI volontaires de première année, d'un même institut de formation, ont été interrogés par la méthode du groupe nominal de consensus. Résultats : Les 7 ESI ayant participé mettent en avant le sommeil comme facteur prédominant en lien avec leur QdV. Le principal domaine qui selon eux favorise une bonne $\mathrm{QdV}$ est le social, regroupant la sphère amicale et l'encadrement de la part des formateurs. Conclusion : Cette étude apporte des pistes de réflexion sur la QdV des ESI, pouvant indiquer des pratiques quotidiennes des équipes pédagogiques ou pour le versant ingénierie de formation de la QdV chez les ESI.

Mots clés : groupe nominal par consensus, qualité de vie, étudiants en soins infirmiers, programmes de formation en soins infirmiers

\begin{abstract}
Context: Nursing students' initial year of training brings about many changes, in their personal, family and academic lives which requires rapid adaptation, which can modify their life balance and ultimately affect their quality of life (QoL). Purpose: The objective was to identify, according to a sample of students because of how it affects them and because they are experts in their own QoL, the factors that could influence their QoL. Method: A group of seven first-year nursing students from the same academic institution, volunteered to participate in the consensus nominal group study. Results: The students highlight sleep as the predominant factor that influences their quality of life. The social dimension of their lives is the one most contributive to maintaining a good QoL, including friendships and support from educators. Conclusion: This study provides valuable insights nursing students' QoL, as well as for their educators and for the curriculum design in nursing programs.
\end{abstract}

Keywords: consensus nominal group, quality of life in training, nursing students, nursing education

\footnotetext{
*Correspondance et offprints: Barbara JOANNOT, 6 rue Bardou, 31200 Toulouse, France.

Mailto: joannot.barbara@gmail.com.
} 


\section{Introduction}

Le parcours de formation professionnelle, en lien avec la vie étudiante qui l'accompagne, est une période de changement pour les personnes concernées. L'interaction de la personne étudiante avec son environnement et ses domaines d'activités, qu'ils soient personnels, familiaux, scolaires ou autres, exigent d'elle une adaptation. En effet, son équilibre occupationnel peut s'en trouver modifié. En élargissant aux dimensions complexes de l'individu, la qualité de vie de l'étudiant est également sujette à modification, comme en témoignent de nombreuses études. Divers facteurs semblent agir sur la qualité de vie $(\mathrm{QdV})$ des étudiants. Quelles informations peuvent nous livrer les principaux concernés? Nous avons interrogé les étudiants en soins infirmiers (ESI) sur cette question vive. À travers un groupe nominal de consensus, nous avons tenté d'identifier les facteurs favorisant la QdV des ESI.

\section{Contexte et problématique}

\section{La notion de qualité de vie}

"Certains parlent de qualité de vie, d'autres de statut de santé, ou de bien-être.» [1]. La QdV est une notion multidimensionnelle $[2,3]$, s'intéressant aux dimensions physiques, mentales et sociales de l'individu dans sa complexité. L'Organisation mondiale de la santé la définit comme «[...] la perception qu'a un individu de sa place dans l'existence, dans le contexte de la culture et de système de valeurs dans lesquels il vit, en relation avec ses objectifs, ses attentes, ses normes et ses inquiétudes. Il s'agit d'un large champ conceptuel, englobant de manière complexe la santé physique de la personne, son état psychologique, son niveau d'indépendance, ses relations sociales, ses croyances personnelles et sa relation avec les spécificités de son environnement» [4].

Ainsi cette notion est associée à une conception holistique de l'individu, prenant appui autant sur des paramètres intrinsèques qu'extrinsèques, qui prend en compte les interactions avec l'environnement et les facteurs sociaux.

\section{La qualité de vie des étudiants}

Nous avons effectué une recherche documentaire ciblée des travaux de recherche dédiés à la qualité de vie des étudiants, en utilisant le moteur de recherche Google scholar $^{\circledR}$ et en interrogeant les bases de données suivantes : Banque de données en santé publique (BDSP), Littérature scientifique en santé (LiSSa), Elesevier-Masson (EM Premium ${ }^{\circledR}$ ) Science Direct ${ }^{\circledR}$, Pascal et Francis ${ }^{\circledR}$ et Cairn $\left.{ }^{\circledR}\right)$. Pour cela, la locution «qualité de vie des étudiants» a été utilisée, suivie d'une sélection via les mots clefs successivement dans le titre, le résumé puis le corps du texte. Les articles incluant «santé» et «formation», ou «bien-être» ont été conservés. Ont été exclus tous les articles non centrés sur les étudiants et traitant de pathologie, de patients, de handicap ou de troubles des apprentissages. Au total, 21 articles abordant la question qui nous intéresse ont été retenus.

Cette revue de la littérature met en avant les différents tests ou échelles utilisées pour l'évaluation de la qualité de vie des étudiants (SF-36, WHOQOL, SQUALES, échelles d'anxiété et de dépression). Les facteurs d'une diminution de la QdV des étudiants sont d'origine interne [5-7] ou externe, tels que l'environnement socioéconomique (finances, hébergement seul ou familial, contacts sociaux et familiaux...), la charge de travail [8-10] et le facteur temps [11]. La diminution de la QdV des étudiants s'exprime à travers différentes formes physiques et psychiques $[12,10]$. Les symptômes observés peuvent être de la fatigue, des troubles du sommeil, de la tristesse, des difficultés à se concentrer, des troubles anxieux, des maux de tête, des douleurs d'estomac ou dans le bas du dos.

Les auteurs énoncent également que l'engagement scolaire ou la réussite dans les études seraient liés à la qualité de vie étudiante [7,13-16]. Ainsi, des propositions visant à favoriser la $\mathrm{QdV}$ des étudiants sont émises par les différentes études, comme la mise en place d'un tutorat entre étudiants [11,17-19], l'importance du recrutement en fonction des expériences et de la motivation [11], la mise en place de services de prévention $[8,17]$.

En France, la mise en place d'un centre national d'appui pour promouvoir la QdV des étudiants et des professionnels de santé était la première recommandation issue de l'enquête nationale menée par Marra [20]. Les autres recommandations, basées sur la prévention, la communication, la formation et l'accompagnement sont actuellement en cours de développement.

\section{Les étudiants en soins infirmiers}

En se basant sur le modèle «Personne-Environnement Occupation Performance», la situation étudiante est définie par les interactions de l'étudiant dans son environnement [21]. Celle-ci est marquée par différents paramètres : le budget, la mobilité, la santé, le bien-être $[10,14,17,22-24]$. La particularité des étudiants en soins infirmiers (ESI) est liée au fait d'être dans un processus de professionnalisation [25]. Comme toutes les professions paramédicales, la formation des ESI s'inscrit actuellement dans le cadre d'une universitarisation graduelle, en visant une harmonisation nationale et européenne [26,27]. Concernant spécifiquement les ESI, leur formation est une source de changement. L'étudiant, en suivant un processus pour devenir soignant, est amené à prendre en compte le mieux-être ou bien-être des patients, pouvant appréhender des situations difficiles liées à la maladie et la souffrance.

\section{La qualité de vie des étudiants en soins infirmiers}

Une récente enquête de la Fédération nationale des étudiants en soins infirmiers (FNESI) sur le bien-être des ESI évoque «une précarité flagrante» et «une santé physique dégradée» des étudiants [19,28]. La FNESI impute cette dégradation de santé à une baisse de l'activité 
physique et à une diminution de la quantité et qualité de sommeil [28]. Des situations difficiles rencontrées en institut de formation en soins infirmiers (IFSI) ou en stage peuvent avoir pour conséquence «stress, angoisse, anxiété, dépression» [28].

Comme évoqué précédemment, la $\mathrm{QdV}$ des étudiants ESI est une notion plus globale que les simples conditions de vie étudiantes. Elle s'intéresse à une vision «méta » des étudiants. L'aspect systémique des interactions de l'étudiant avec le milieu académique et le milieu de soin est souligné par le rapport de Marra [20].

Hors de France, Yanxiang et al. soulignent l'importance de la prise en compte des facteurs de QdV des ESI par les formateurs [29]. Une démarche proactive de recueil de données auprès des étudiants permet d'être à l'écoute et d'obtenir des éléments liés à la singularité des étudiants de l'institut. De telles informations constituent des bons indicateurs pouvant servir de support au Conseil de la vie étudiante. De façon encore plus méthodique et systématique, la démarche qualité, vecteur de changement, est maintenant engagée dans les instituts de formation. Elle place l'étudiant au centre du dispositif et fournit de nombreux indicateurs de ce type, qui permettent de réfléchir à la ré-ingénierie des projets pédagogiques. D'une façon générale, l'implication des étudiants dans une démarche de ce type permet de les habituer à intégrer la qualité dans leur processus de professionnalisation, une telle dimension étant d'ailleurs reprise et formalisée au travers de la compétence $7 \mathrm{du}$ référentiel de formation [26].

Dans ce contexte, nous avons entrepris un travail visant à identifier quels étaient, du point de vue des étudiants eux-mêmes, considérés comme expert de leur qualité de vie, les facteurs susceptibles d'influencer cette dernière, au regard d'une approche multidimensionnelle.

\section{Méthodes}

\section{Le choix méthodologique du recours à un groupe nominal}

Le groupe nominal est l'une des méthodes d'élaboration de consensus formalisé [30]. Son intérêt est de pouvoir obtenir puis hiérarchiser des informations. L'échantillon est composé de 5 à 15 personnes et d'un animateur, expert ou non dans le domaine. "Les interactions se font essentiellement entre le coordinateur et chaque membre du groupe pour éviter les phénomènes de dominance» [30].

L'objectif poursuivi par le recours à cet outil est d' «analyser un problème, explorer un champ de connaissance, faire une synthèse globale d'une question». En l'occurrence, l'objet de l'enquête dont nous rapportons les résultats concerne la qualité de vie des ESI [30]. Ainsi, nous utilisons une méthode descriptive, c'est-à-dire que nous nous attachons à décrire afin de «transmettre une information précise complète et exacte» [31].

\section{Sélection des participants}

Les étudiants ayant participé à l'enquête étaient tous volontaires. L'échantillon était composé de sept ESI en première année de formation au sein du même IFSI. Il comportait deux hommes et cinq femmes, d'âge moyen 26,7 ans (écart type : 7 ans), légèrement plus élevé que celui de l'ensemble de la promotion $(25+/-8,9$ ans $)$. Les étudiants ont tous déjà effectué un stage.

\section{Déroulement du groupe nominal de consensus}

\section{Principes généraux}

L'animateur, étudiante en formation cadre de santé, était extérieur à l'équipe pédagogique, de manière à ne pas favoriser le biais de désirabilité sociale. Les participants ont été invités à se regroupés dans une salle, au sein de laquelle avait été au préalable préparé le matériel nécessaire (paperboard avec des feutres, feuilles individuelles avec les consignes).

Le projet a été expliqué aux participants par l'animateur, qui s'est ensuite montré disponible pour répondre à toutes questions sur le déroulement ou l'engagement des étudiants. Dans le respect de la dimension éthique de la démarche, le respect de l'anonymat des étudiants a été précisé et le consentement libre et éclairé des participants a été recueilli avant de débuter le groupe nominal. Il a été rappelé que chaque étudiant, expert de sa QdV, est «responsable de son dire et de son faire» [32] mais la confidentialité des échanges a été garantie, l'engagement étant pris que seraient constituées comme seules traces les documents validés par l'ensemble du groupe (tableaux de classement, rangs et explications des facteurs).

\section{Les étapes opérationnelles}

Les étapes du groupe nominal de consensus, tel qu'il a été organisé auprès des ESI, ont respecté les recommandations publiées dans la littérature [30,33] :

- explication du déroulé aux participants. Il est précisé, qu'il n'y a pas de bonnes ni de mauvaises réponses. Tous les participants s'engagent dans le respect d'autrui, sans jugement, dans un climat de confiance;

- distribution d'une feuille par l'animateur (étudiante cadre de santé), avec la question écrite: «En tant qu'étudiant (e), quels sont les facteurs favorisant votre qualité de vie?». Les étudiants répondent par quatre mots, individuellement. Ils ne peuvent pas échanger entre eux;

- énoncé des idées : l'animateur demande à chaque participant, à tour de rôle, de communiquer la première réponse inscrite sur sa feuille. On ne recueille aucune critique ou commentaire de la part des participants ou de l'animateur. Si un mot a déjà été donné, le participant énonce son mot suivant, jusqu'à épuisement de tous les mots ;

- clarification de chaque mot ou idée donnés par le groupe, sans que la personne ayant énoncé le mot ne s'exprime. Une discussion est engagée entre les participants qui se mettent en accord sur une explication de chaque item. Après accord entre les participants, l'animateur écrit l'explication sur un tableau. Le temps d'explication entre chaque item est réparti ; 
Tableau I. Synthèse du classement des facteurs énoncés par les étudiants en soins infirmiers.

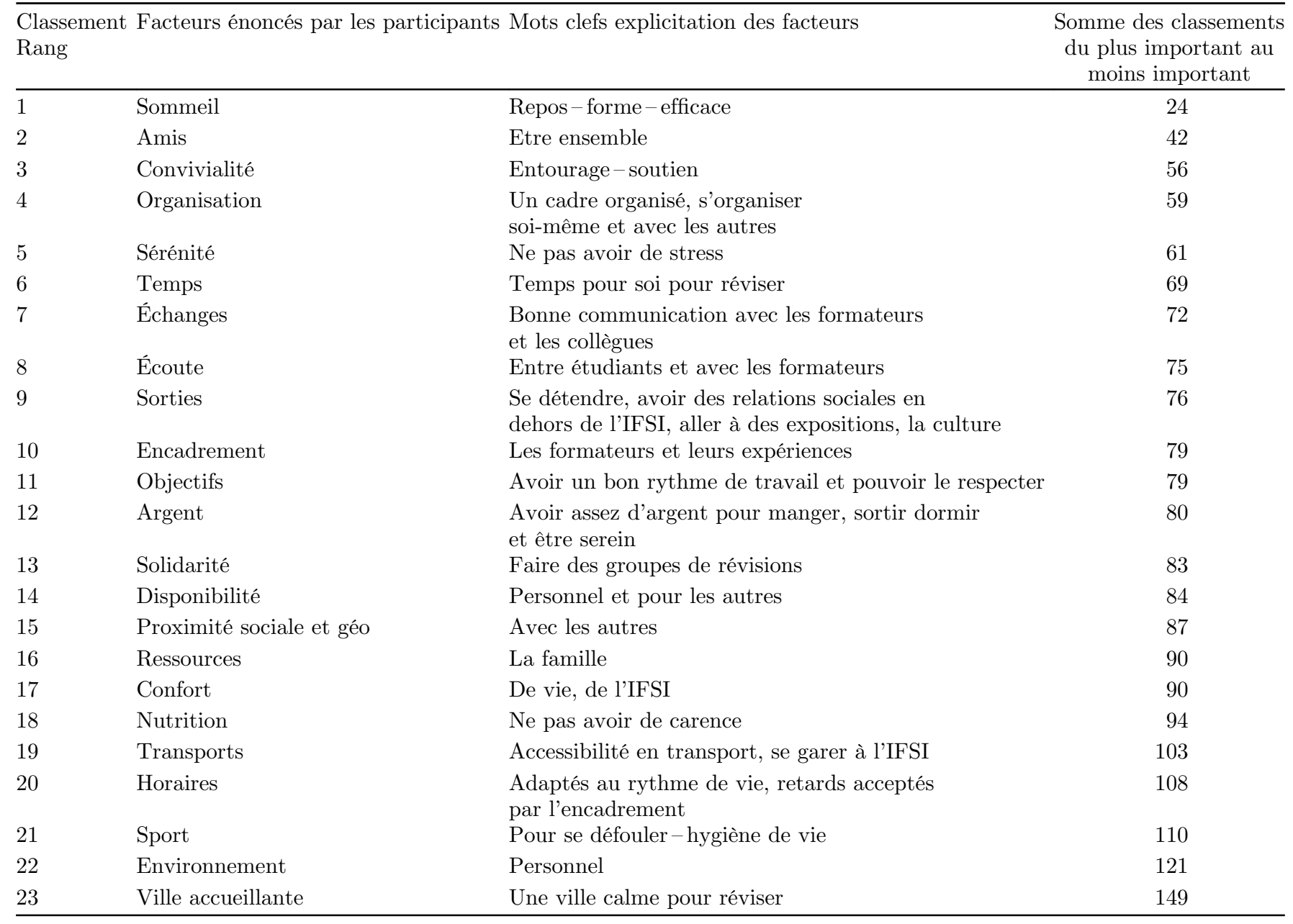

IFSI : institut de formation en soins infirmiers.

- cotation individuelle de chaque idée par les participants sur la feuille, chacun d'entre eux les classant par ordre d'importance;

- catégorisation des items en différents domaines, effectuée dans le cadre d'un échange en groupe puis, une fois les domaines déterminés, classement par domaine;

- synthèse des classements de chaque item et de chaque domaine par ordre d'importance, effectuée par l'animateur.

\section{Résultats}

Une synthèse des classements des énoncés formulés par les sept étudiants est présentée dans le tableau I, qui mentionne les scores obtenus par la somme des rangs de chaque facteur coté individuellement, ce qui permet un classement du plus important au moins important. Les étudiants placent dans les dix premiers facteurs le sommeil, puis les amis, la convivialité, l'organisation, la sérénité, le temps, les échanges, l'écoute, les sorties, l'encadrement.
Globalement, en observant l'explicitation des mots par les étudiants, nous relevons que les facteurs favorisant leur QdV sont principalement centrés sur eux et sur la relation à l'autre (sommeil, amis convivialité, temps, échange, écoute, sorties). Cet élément est repris dans le classement par domaine. (Tableau II).

\section{Discussion}

Réalisée avec un échantillon de sept étudiants volontaires, la démarche de consensus formalisé développée avec des ESI, dont nous avons rapportés les résultats, a permis d'apporter des données singulières. Les étudiants n'ont pas été questionnés sur les améliorations qu'ils souhaiteraient observer ou solutions qu'ils proposeraient. À cet égard, les recommandations faites dans le rapport national en cours d'application apportent des éléments opérationnels, tandis que notre étude informe sur les facteurs que priorisent les étudiants dans leur quotidien.

Les éléments déterminés par le travail corroborent les données de la littérature concernant les étudiants. Une métanalyse croisant 13 articles différents met en avant 
Tableau II. Classement des domaines regroupant les facteurs qui favorisent la qualité de vie des étudiants en soins infirmiers.

\begin{tabular}{|c|c|c|}
\hline Classement & Domaines & $\begin{array}{c}\text { Pondération inversée } \\
\text { du classement }\end{array}$ \\
\hline 1 & $\begin{array}{l}\text { Social } \\
\text { (sorties, amis, écoute, proximité, solidarité, convivialité, } \\
\text { échanges, encadrement) }\end{array}$ & 13 \\
\hline 2 & $\begin{array}{l}\text { Moyens } \\
\text { (disponibilité, argent, ressources, transport, temps, horaire, objectif, } \\
\text { sérénité, organisation) }\end{array}$ & 15 \\
\hline 3 & $\begin{array}{l}\text { Hygiène de vie } \\
\text { (sport, nutrition, sommeil) }\end{array}$ & 15 \\
\hline 4 & $\begin{array}{l}\text { Environnement } \\
\text { (ville accueillante, confort, environnement) }\end{array}$ & 27 \\
\hline
\end{tabular}

qu'il existe une association négative entre le stress et la QdV chez les étudiants universitaires, traduite par une détérioration de différents aspects relatifs à la santé physique et mentale. «Les facteurs pointés sont le burn out, les troubles du sommeil et la dépression. » [34]. En accord avec ces données, le sommeil est le premier facteur mis en avant lors du groupe nominal de consensus. Cette même revue systématique rapporte une étude de Kleiveland et al. [35], mettant en avant l'association négative entre «un haut niveau de stress et la qualité de vie chez les étudiants infirmiers.» [34]. En élargissant aux étudiants de la première année commune aux études de santé (PACES), deux éléments ressortent : le stress académique et le stress lié aux relations aux proches. Le stress académique est lié à la charge de travail et le stress en lien avec les relations concerne le contexte stressant d'autonomisation des étudiants vis-à-vis de leurs proches [10]. Dans le cadre de notre travail recourant au groupe nominal de consensus, l'échantillon n'était pas composé d'étudiants en PACES ; cependant les relations sociales sont mises en avant comme facteurs favorisant la QdV. Hors du champ médical, «les stresseurs mineurs des étudiants de grandes écoles concernent principalement la santé, les relations interpersonnelles et la gestion du temps.» [8]. Dans notre étude, les étudiants ont priorisé le domaine social comme facteur favorisant leur QdV ; la famille apparaît en ressource en $19^{\mathrm{e}}$ position. L'organisation (en quatrième rang) et le temps (en sixième rang) sont également évoqués comme éléments pouvant favoriser la QdV des ESI.

Dans une visée d'accompagnement, la prise en compte de ces différents facteurs apparaît essentielle. Marra énonce par exemple que «la gestion du stress est un élément qui ne peut être négligé pour un apprentissage performant » [36]. Une étude par une approche centrée sur la personne a montré la relation entre «burn out », profil de QdV et motivation académique [37]. Plus l'étudiant a un niveau de burn out bas, plus sa QdV est élevée et plus sa motivation intrinsèque et son auto-contrôle sont élevés [37] Le domaine social mis en avant par les ESI dans le groupe nominal n'est pas spécifique aux ESI ou aux étudiants français. Une étude auprès des étudiants en médecine en Iran encourage les programmes et initiatives permettant aux étudiants à d' «être physiquement et socialement actifs $\gg[38]$.

\section{Conclusion}

L'étude recourant à un groupe nominal, que nous avons conduite, fournit des éléments qui, mis en perspective avec ceux déjà disponibles dans la littérature, soulignent la complexité et la multidimension de la $\mathrm{QdV}$ chez les ESI. Les leviers dont dispose le formateur sont minces face à cette problématique complexe. Outre la posture et la relation d'aide pédagogique, la bienveillance, l'écoute et une attention à l'organisation font bien partie des conditions sur lesquelles le formateur peut agir dans sa pratique quotidienne, bien qu'elles ne soient pas les seuls facteurs d'une QdV favorable chez les ESI.

En tant que démarche d'élaboration de consensus formalisé, le groupe nominal, simple à effectuer, rapide et informatif, semble pouvoir être transférable à d'autres sujets, centrés sur les étudiants ou les membres d'une équipe de professionnels de santé, notamment dans le champ de travaux de recherche exploratoire.

\section{Contributions}

Barbara Joannot a réalisé le groupe nominal de consensus. Yannick Dauge a apporté une contribution visant à garantir la fidélité du contexte professionnel en soins infirmiers. Jean-Louis Boutte a validé la méthodologie de recherche.

\section{Liens d'intérêts}

Aucun des auteurs ne déclare de conflit d'intérêts en lien avec le contenu de cet article.

\section{Comité éthique}

Non sollicitée.

\section{Références}

1. Leplège A. De la santé perceptuelle à la qualité de vie: évolution conceptuelle et enjeux epistémologiques. In: Fabien B. La qualité de vie. Rennes : Presses Universitaires de Rennes: Collection Psychologie, 2013:15-23. 
2. Boini S. Déterminants de la qualité de vie liée à la santé et de son évolution dans une population à priori saine. Thèse pour le diplôme d'état de docteur en médecine. Nancy : Université de Lorraine, 2005.

3. Leplège A. Les mesures de la qualité de vie. Paris: Presses Universitaire de France, 1999.

4. Organisation Mondiale de la Santé. World Health Organization. 1994 [Online] Disponible sur : http://www.who.int/fr/.

5. Neveu D, Doron J, Visier L, Boiché J, Trouillet R, Dujols P, et al. Students perceived stress in academic programs: Consequences for its management. Rev Epidemiol Sante Publique 2012;60:255-64.

6. Haag P. Anxiété-trait, stress perçu et symptômes somatiques chez les doctorants. Mémoire de master 1. Paris: Université Paris Ouest Nanterre, 2012.

7. Louvel A. La promotion de la santé des étudiants est prise en compte par la région Rhône-Alpes. Entretien avec Thierry Philippe. La santé de l'Homme 2008;393:41-2.

8. Strenna L, Chahraoui K, Reveillère C. Tracas quotidiens des étudiants de première année de grandes écoles : liens avec la santé mentale perçue et la qualité de vie. Ann Med Psychol (Paris) 2014;172:369-75.

9. Terral C. Éducation pour la santé des étudiants. Quelques règles esssentielles. La santé de l'Homme. 2008;393:34-7.

10. Bonnaud-Antignac A, Tessier P, Quere M, Guihard E, Hardoin J, Nazih-Sanderson F, et al. Stress, qualité de vie et santé des étudiants. Suivi d'une cohorte en première année commune d'étude en santé (PACES). J Ther Comport Cogn $2015 ; 25: 58-65$.

11. Gueguen C, Moulin L. Bien-être et stratégies d'apprentissage des étudiants en première année commune aux études de santé. Thèse pour le diplôme d'état de docteur en médecine. Université d'Angers, 2015.

12. Migeot V, Ingrand I, Defossez G, Salardaine F, Lahorgue M, Poupin C. Comportement de santé des étudiants d'IUT de l'université de Poitiers. Santé Publique 2006;18(2):195-205.

13. Galap C. Créer des centres de santé pour les étudiants. La santé de l'Homme 2008;393:21-2.

14. Brault-Labbé A, Dubé L. Engagement scolaire, bien-être personnel et autodétermination chez les étudiants à l'université.Can J Behav Sci 2010;2:80-92.

15. Revillière C, Nandrino JL, Sailly F, Mercier C, Moreel V. Études des tracas quotidiens des étudiants: liens avec la santé perçue. Ann Med Psychol (Paris) 2001;159:460-5.

16. Blanchet D. Analyse du facteur de l'anxiété vécue chez les étudiants québéquois au niveau collégial. Thèse pour le Doctorat d'Université en Psychologie. Université de Sherbrooke, 2011.

17. Amara ME, Baumann M, Pelt V, Guillaume J-F, Ionescu I. Student's Quality of Life and Employability Skills : SQALES un dispositif et un instrument au service des universités. Revista de Cercetare si inventie sociala. 2010;28:97-114.

18. Diot P. Études de médecine en France: Comment sortir d'un si profond malaise? Presse Med 2017;46:1119-21.

19. Lamaurt F, Estryn-Behar M, Le moel R, Chrétien T, Mathieu B. Enquête sur le vécu et les comportements des étudiants en soins infirmiers. Rech Soins Infirm 2011;2:44-59.

20. Marra D. Rapport au Ministre de la santé et des solidarités et au Ministre de l'enseignement supérieur, de la recherche et de l'innovation sur la qualité de vie des étudiants en santé. 2018
[On-line] Disponible sur: https://solidarites-sante.gouv.fr/ IMG/pdf/180403_-_rapport_dr_donata_mara.pdf.

21. Margot-Cattin I. Le modèle Personne-EnvironnementOccupation-Performance (PEOP). In: Morel-Bracq MC. Les modèles conceptuels en ergothérapie. Louvain-laNeuve: De Boeck, 2017:63-72.

22. Felouzis G. La condition étudiante : sociologie des étudiants et de l'Université. Paris : Presses Universitaires de France, 2001.

23. Gaillard R, Rexand-Gallais F. La condition étudiante à l'épreuve du territoire? Rennes: Presses Universitaires de Rennes, 2017.

24. Strenna L. Études des tracas quotidiens des étudiants de Grandes Écoles : lien avec la santé perçue, la qualité de vie et l'importance de prendre en compte de l'influence des traits de personnalité et de l'estime de soi. Thèse pour le Doctorat d'université. Dijon: Université de Bourgogne, 2011.

25. Wittorski R. La professionnalisation. Savoirs 2008;2:9-36.

26. Ministère de la santé et des sports. Arrêté du 31 juillet 2009 relatif au diplôme d'État d'infirmier. Bulletin Officiel Santé-Protection sociale-Solidarités 2009/7:246-383.

27. Ministère des solidarités et de la santé-Ministère de l'enseignement supérieur. Étudiants en soins infirmiers, un pas décisif vers l'université. Dossier de presse. 2018 [Online] Disponible sur: https://solidarites-sante.gouv.fr/ IMG/pdf/180705_-_dp_-_universitarisation_des_infir miers.pdf.

28. Fédération nationale des étudiants en soins infirmiers. [Online]; 2017 [cited 2017 novembre 5]. Available from: http://fnesi.org/communique-de-presse/18-septembre2017-dossier-de-presse-mal-etre-des-etudiant-e-s-en-soinsinfirmiers-il-est-temps-dagir/.

29. Yanxiang C, Min X, Xiuyun L. Psychometric properties of an instrument to measure nursing' quality of life. Nurse Educ Today 2005;35:e1-5.

30. Bourée F, Michel P, Salmi LR. Méthodes de consensus: revue des méthodes originales et de leurs grandes variantes utilisées en santé publique. Rev Epidemiol Sante Publique 2008;56:415-23.

31. Martel F. Méthodologie: la méthode descriptive, son fondement théorique. Rech Soins Infirm 1988;15:56-8.

32. Ricoeur P. Réflexion faite, autobiographie intellectuelle. Paris : Édition Esprit, 1995.

33. Letrilliart L, Venmeerbeerk M. À la recherche du consensus : Quelle méthode utiliser? Exercer 2011;22:170-7.

34. Ribeiro IJS, Pereira R, Freire IV, De Oliveira BG, Casotti CA, Boery EN. Stress and quality of life among university students: A systematic literature review. Health Professions Education 2017;4:70-7.

35. Kleiveland B, Natvig G, Jepsen R. Stress, sense of coherence and quality of life among Norwegian nurse students after period of clinical practice. Peer J 2015;3:e1286.

36. Marra D. Performance, apprentissage et santé des étudiants. Les tribunes de la santé 2012;2:51-6.

37. Lyndon MP, Henning MA, Alyami H, Krishna S, Zeng I, Yu TC, et al. Burnout, quality of life, motivation and academic achievement among medical students: A person-oriented approach. Perspect Med Educ 2017;6:108-14.

38. Jamali A, Tofangchiha S, Jamali R, Nedjat S, Jan D, Narimani A, et al. Medical students' health-related quality of life: Roles of social and behavioural factors. Med Educ 2013;47:1001-12.

Citation de l'article : Joannot B., Dauge Y., Boutte J.-L. Utilisation d'un groupe nominal de consensus en institut de formation en soins infirmiers : un éclairage sur la qualité de vie des étudiants. Pédagogie Médicale, https://doi.org/10.1051/pmed/2019023 